\title{
The relationship between survival gastric cancer patients and neutrophil-to-lymphocyte ratio and platelet-to-lymphocyte ratio diagnosed before surgery
}

\section{Sareh Mohammadi}

Shahrekord University of Medical Science

\section{Zeinab Veisi}

Shahrekord University of Medical Science

Mohammad Moazeni

Shahrekord University of Medical Science

\section{Soleiman Kheiri}

Shahrekord University of Medical Science

\section{Zahra Lorigooini}

Shahrekord University of Medical Science

Rohollah masomi ( $\square$ masomirohollah@gmail.com )

Shahrekord University of Medical Science https://orcid.org/0000-0003-4439-6214

\section{Research}

Keywords: Gastric Cancer, Neutrophil, Lymphocyte, Platelet

Posted Date: November 6th, 2020

DOl: https://doi.org/10.21203/rs.3.rs-100927/v1

License: (c) (i) This work is licensed under a Creative Commons Attribution 4.0 International License.

Read Full License 


\section{Abstract}

Aim: This study aims to investigate the relationship of two prognostic factors NLR and PLR to patient survival in gastric cancer patients diagnosed before surgery.

Background: Due to the high prevalence of gastric cancer and the lack of appropriate prognostic factors, most patients are diagnosed at advanced stages of the disease. Therefore, this study aimed to determine the appropriate prognostic factor investigated investigate the relationship of neutrophil-to-lymphocyte ratio (NLR) and platelet-to-lymphocyte ratio (PLR) in gastric cancer patients.

Methods: This retrospective study was performed on all gastric cancer patients referred to Kashani Hospital of Shahrekord (Chaharmahal and Bakhtiari province) in 2011-2016. Demographic and biological variables such as NLR and PLR were evaluated. Patient survival was calculated by subtracting the date at disease diagnosis (using endoscopy) from the date at death and expressed in month. Data was analyzed by SPSS software.

Results: The mean age of patients was $63.79 \pm 15.03$ (range: $10-92$ ) years. $76 \%$ of patients were male and the rest were female. Only NLR had significant effect on survival $(P<0.05)$ and PLR had no significant effect on survival $(P>0.05)$.

Conclusion: With the increase in NLR by one point, the risk of death in gastric cancer patients increased by $18 \%$. In other words, with increase in NLR, survival rate of gastric cancer after surgery significantly decreased. It is suggested that further studies be conducted with a more statistical population to ensure the reproducibility of the results of the present study.

\section{Introduction}

Gastric cancer is one of the leading causes of cancer deaths worldwide. Over a million cases of gastric cancer are diagnosed worldwide each year. Gastric cancer is the fifth leading cancer worldwide and the third leading cause of cancer deaths with an estimate of approximately 783,000 deaths in 2018 [1]. About $75 \%$ of new cases of this cancer occur in developing countries. In Iran, more than 50,000 new cases of cancer are reported each year [2]. There are several factors that contribute to cancer. Chronic inflammatory conditions caused by genetic mutations, autoimmune diseases, and exposure to environmental factors can increase the risk of developing cancer. Previous studies have shown the association of inflammation and inflammatory reactions with atherosclerosis and thrombosis as well as with heart disease in renal disease patients [3-5]. Regarding the association of these factors with cancer, epidemiological studies have shown that more than $25 \%$ of cancer deaths are due to chronic inflammation [6]. Numerous studies have shown that inflammation leads to the continuation and progression of cancer as well as complete changes in malignancies, such as alterations in tumor tissue status, angiogenesis, and metastasis, preventing and suppressing the anti-cancer immune response [7]. Although the systemic inflammatory response activity involves the body's various organs, the most important parameter to measure inflammation is the counts of white blood cells and acute-phase 
proteins. Specifically, white blood cell counts such as neutrophils, lymphocytes, and platelets, blood Creactive protein (CRP) levels and albumin as well as neutrophil-lymphocyte ratio (NLR) and plateletlymphocyte ratio (PLR) have been used as inflammatory parameters [3]. There are many reports that high density of neutrophils may stimulate tumor growth and metastasis and impair the immune system's antitumor response by suppressing lymphocytes. These observations suggest that high NLR in peripheral blood of cancer patients may lead to tumor progression [8-10]. However, little is known about the importance of NLR for diagnosis of gastric cancer $[11,12]$. Thrombocytosis occurs due to the stimulation of megakaryocytes by inflammatory cytokines, and high platelet count is known as an inflammatory marker. High platelet count is another parameter that is helpful in predicting patients' prognosis. Increased platelet-to-lymphocyte ratio has been reported as an independent factor in decreasing the life expectancy of patients with colon and pancreatic cancer $[13,14]$. Neutrophils, however, are a stronger factor in inflammation and survival in cancer patients than thrombocytosis [15]. Given the systemic nature of gastric cancer and poor prognosis, treatment despite surgery, radiotherapy, and chemotherapy, information about the diagnostic factors that predict the course of the disease is highly effective to adopt better treatments. Although the incidence of this cancer appears to be decreasing, due to the slow nature of the disease, it is very difficult to diagnose it so that in Iran, nearly $50 \%$ of the cases are diagnosed in the advanced stages and 5-year survival is very low $[2,16]$. In Chaharmahal and Bakhtiari province, gastric cancer is highly prevalent and over $50 \%$ of the patients are diagnosed in the advanced stages. Knowledge about the patient's diagnostic factors makes the planning of treatment for patients more accurate and helps to use standard treatments of choice for these patients. Given that NLR and PLR in many cancers are predictors of disease progression, invasion and even lower treatment response, these measures need to be evaluated in gastric cancer patients so that their association with the disease prognosis and patient survival will be established. Therefore, the aim of the present study was to investigate the relationship of two prognostic factors NLR and PLR to patient survival in gastric cancer patients diagnosed before surgery.

\section{Methods}

This is a retrospective study. All patients referred to Kashani Hospital from 21 March 2011 to 19 March 2016 for treatment of gastric cancer and filed in the hospital were retrospectively studied. Patients whose disease was at a treatable stage were included. The exclusion criteria included suffering from more than one malignancy, underlying systemic disease such as diabetes and infectious comorbidities. To determine the stage of the disease, the Staging System and the American Joint Committee on Cancer (AJCC Staging Manual) method were used as follows: Early Stage: Mucosal and submucosal involvement without involvement of local lymph nodes (T1, T2, N0). Locally advanced Stage (T3, T4, N+). Gastric metastasis with any distant metastasis (M1). The survival duration of patients after surgery was evaluated. The NLR and PLR were calculated in patients undergoing surgery before the surgery. The correlation between these ratios and postoperative patient survival was investigated. The studied variables were of demographic and biological types. Demographic variables included patient's age at diagnosis, gender, and place of residence. Biological variables included history of chronic gastrointestinal 
disease, history of cancer in close or otherwise relatives, NLR, PLR, tobacco smoking, type of treatment done, degree of tumor differentiation, disease progression, type of histopathology, symptoms of the disease at diagnosis, and literacy. The data was collected from the medical records of patients and the latest physical conditions of the patients were investigated by telephone contact and registered in the prepared checklists. Patient survival was calculated by subtracting the date of diagnosis (using endoscopy) from the date of death or being censored and expressed in month. In this study survived patients at the completion of the study and missing persons at follow-up were censored, and for all those who died during follow-up, the cause of death was registered. Data was analyzed using SPSS software. Kaplan-Meier estimator was used to evaluate cumulative survival rate and Cox regression model was used to compare survival rates and factors affecting survival. $P<0.05$ was considered significance level.

\section{Results}

The aim of this study was to investigate the relationship of the prognostic measures NLR and PLR to survival in gastric cancer patients diagnosed before surgery in Shahrekord. To this end, a total of 100 gastric cancer patients who had undergone surgery were enrolled in the study. The age range of the patients was 10-92 (mean: $63.79 \pm 15.03$ ) years. $76(76 \%)$ patients were male and the rest were female. Other characteristics of patients such as education level, place of residence, smoking status, duration of being smoker, presence of gastrointestinal disease, and duration of chronic gastrointestinal disease are listed in Table 1. The pathology test results confirmed the presence of a cancerous tumor for $99(99 \%)$ patients with only one negative pathology test result. $5 \%$ of patients had no gastrointestinal disease and $46 \%$ had gastrointestinal disease. The longest duration of disease was 3 years $(n=15)$, followed by 2 years $(n=10)$ and four years $(n=9)$. The duration of chronic gastrointestinal disease before cancer diagnosis ranged from one year to 12 years (mean: $3.3 \pm 2.06$ years). The mean values of inflammatory markers are shown in Table 2. The mean NLR was $3.37 \pm 2.78$ and the mean PLR was 147.13 \pm 78.93 . Patient survival after surgery until death or last follow-up ranged from 14 days to 1892 days (mean: $548.56 \pm 405.90$ days). The mean survival in the survived and died patients was $506.57 \pm 343.29$ days and $559.72 \pm 422.28$ days, respectively Table 3 . The Kaplan-Meier survival function for our patients is shown in Fig. 1. The Kaplan-Meier cumulative hazard function for the patients is also shown in Fig. 2. The estimation of survival time by Kaplan-Meier method is presented in Table 4. The correlation coefficient $(r)$ between NLR and PLR was $0.556(P<0.001)$, indicating a significant direct correlation between the two indices. Regarding the factors affecting patient survival in univariate model, Cox proportional hazard for both NLR and PLR had significant effect on survival. After the simultaneous inclusion of both NLR and PLR, only the NLR variable had a significant effect on patient survival Table 5. The results indicated that in the presence of the variable NLR, the variable PLR had no effect on survival. In other words, the main variable affecting survival of patients was NLR. With the increase of NLR by one point, the survival rate of gastric cancer patients after surgery decreased by $18 \%$. More clearly, the survival rate of gastric cancer patients after surgery decreased significantly with increasing NLR. Cox regression model showed no association between age, gender, smoking, education, place of residence, duration of chronic gastrointestinal disease and survival of patients. 


\section{Discussion}

Systemic inflammatory response activity in a variety of conditions, including cancer, can affect different organs of the body. The most important parameter to measure inflammation is the counts of white blood cells and acute-phase proteins. Specifically, white blood cell count such as neutrophils, lymphocytes and platelets, and blood CRP levels and albumin as well as NLR and PLR have been used as inflammation parameters [3]. In the present study, the relationship of two prognostic factors NLR and PLR to survival in gastric cancer patients diagnosed before surgery in Shahrekord was investigated. The mean age of the studied patients was $63.79 \pm 15.03$ (range: $10-92$ ) years. $76 \%$ of patients were male and the rest were female. In the study of Biglarian et al. (2009) on postoperative survival in patients with gastric cancer, it was reported that $71.6 \%$ of patients were male with the mean age of 59.39 years and the rest $(28.6 \%)$ were female with the mean age of 56.22 years [17]. In the study of Moghimi Dehkordi et al. entitled Modeling Survival Analysis Using Cox Model in Gastric Cancer Patients, $68.6 \%$ of patients were male. The mean age of the patients was 58.4 years [18]. The results of the present study are consistent with the above studies regarding gender and age distribution in gastric cancer patients. In the study of Biglari et al. (2009), the mean and median survival of patients with gastric cancer were 32.56 and 28.3 months, respectively, and one, two, three, four and five-year survival rates were obtained $0.78,0.53,0.40,0.32$ and 0.15 , respectively. Cox proportional hazard showed that age at diagnosis, family history, and tumor size were significantly correlated with patients' longevity [19]. In this study, Cox regression model showed no relationship between age, gender, smoking, education, place of residence, duration of chronic gastrointestinal disease and survival of patients. The mean NLR and PLR were $3.37 \pm 2.78$ and $147.13 \pm$ 78.93, respectively. The correlation coefficient $(r)$ between NLR and PLR was $0.556(P<0.001)$, indicating a significant and direct correlation between the two indices. In evaluating the factors affecting survival in patients in univariate model, the Cox proportional hazard of both NLR and PLR were found to have a significant effect on survival. In the present study, $54 \%$ of the studied patients had no gastrointestinal disease and $46 \%$ had gastrointestinal disease. The maximum duration of the disease was 3 years $(n=$ $15)$, followed by 2 years $(n=10)$ and 4 years $(n=9)$. Patient survival after surgery until death or last follow-up ranged from 14 days to 1892 days (mean: $548.56 \pm 405.90$ days). The mean survival in the survived and died patients was $506.57 \pm 343.29$ days and $559.72 \pm 422.28$ days, respectively. After inclusion of both NLR and PLR, only NLR was found to have a significant effect on patient survival. The results indicated that in the presence of NLR, PLR had no effect on survival. In other words, the main variable affecting survival of patients was NLR. With an increase in NLR by one point, the risk of death in gastric cancer patients increased by $18 \%$. In other words, with an increase in NLR, the survival rate of gastric cancer patients after surgery significantly decreased. Various studies have reported that NLR in patients with gastric cancer is prognostically valuable before surgery and higher preoperative NLR, as a strong independent factor, is associated with poorer prognosis in gastric cancer patients $[15,20]$. Other studies have also reported that comparably higher NLR was associated with lower survival in patients with malignant pleural mesothelioma [21]. There are many reports that high density of neutrophils may stimulate tumor growth and metastasis and impair the immune system's antitumor response by suppressing lymphocytes. These observations suggest that high NLR in peripheral blood of cancer 
patients may lead to tumor progression [8-10]. Regarding studies on the prognostic importance of PLR in cancer patients, Smith et al. (2008) in a study in patients with pancreatic adenocarcinoma reported that the PLR ratio was prognostically important before surgery,and is a more reliable marker for prognosis of cancer than lymphocyte count alone [13]. Gu et al., (2017) also reported that a high PLR was significantly associated with overall survival but not with disease-free survival. High PLR also significantly predicted a poor and low overall survival in whites, chemotherapy recipients, and Patients with advanced cancer. In addition, the PLR over 160 was prognostically appropriate. High PLR was also associated with lymph node metastasis and CEA levels in gastric cancer [22]. In other studies, increased PLR has been reported as an independent factor for reduced longevity of patients with colon and pancreatic cancer $[13,14]$. However, a study by Matowicka-Karna et al. (2013) showed that gastric cancer progression was associated with a decrease in the levels of IL- 6 and IL-23, with no association with platelet count or morphological features [23]. It is suggested to investigate the relationship of the levels of the two ILs to NLR and PLR with respect to survival in gastric cancer patients in additional studies. Dogan et al. (2015) reported that in patients with metastatic gastric cancer, PLR $>160$ or NLR $\geq 2.5$ was significantly associated with poorer survival in these patients [24]. He et al. also comparatively studied NLR and PLR and reported that NLR was more efficient than PLR as a prognostic marker for colon cancer [25_ENREF_25]. The results of the present study also showed that NLR, as compared to PLR, was the main factor associated with the survival of gastric cancer patients. Therefore, according to the results of this study, the main variable affecting the survival of gastric cancer patients is NLR that can be used as a prognostic factor related to the survival of these patients.

\section{Conclusion}

Our results showed that PLR had no effect on survival in the presence of NLR. In other words, the main variable affecting the survival of patients was NLR. With increase in NLR by one point, the risk of death in gastric cancer patients increased by $18 \%$. In other words, with the increase of NLR by one point, the survival rate of gastric cancer patients after surgery significantly decreased.

\section{Declarations}

\section{Ethics approval and consent to participate}

All stages of experimentation were carried out in accordance with the regulations of the ethical committee of Shahrekord University of Medical sciences (Ethics code: IR.SKUMS.REC.1395.117).

\section{Consent to publish}

Not applicable.

\section{Availability of data and materials}


The datasets used and/or analyzed during the current study are available from the corresponding author on reasonable request.

\section{Competing interests}

The authors declare that they have no competing interests.

\section{Funding}

This study was funded by Research and Technology Deputy of Shahrekord University of Medical Sciences (Grant no: 2156). The funders have no role in the design of the study, data collection, analysis, interpretation of data and in writing the manuscript.

\section{Authors' contributions}

RM was responsible for the conception of the study and participated in the in design and worked on the draft of manuscript. SM, MM and SKh designed, conducted and interpreted the research outcome of the study, performed the statistical analysis and worked on the draft of manuscript. SM and ZV performed the assay and made contribution to the revision of the draft manuscript. ZV worked on the role of the patients and experiments in the study. All authors read and approved the final manuscript. SM, ZL and RM prepared the manuscript. Before submission all the authors have checked and approved the final manuscript.

\section{Acknowledgements}

This study was derived from the MD thesis written by Zainab Veisi and funded by Shahrkord University of Medical Sciences. The authors of this study appreciate Kashani Hospital, Shahrekord University of Medical Sciences, Shahrekord, Iran and all participated in this study.

\section{References}

1. Rawla P, Barsouk A. Epidemiology of gastric cancer: global trends, risk factors and prevention. Przeglad gastroenterologiczny. 2019; 14 (1): 26 - 38.

2. Hajizadeh E, Fatemi SR. Application of Bayes method in determining of the risk factors on the survival rate of gastric cancer patients. Koomesh. 2010; 11(2): 129 - 32.

3. Shiva A, Arab S. The effect of inflammation on presence of cancer. J of clin exc. 2015; 4(1): 57-67.

4. Rasouli M, Nesarhosseini V, Kiasari AM, Arab S, Shariati R, Kazemi D, et al. The multiplicative interactions of leukocyte counts with some other risk factors enhance the prognostic value for coronary artery disease. Cardiology journal. 2011; 18(3): 246 - 53.

5. Arab S, Khazaai H, Hambali, Z, Ahmad Z. Homocysteine and malondialdeyde (MDA) levels associated with the occurrence of cardiovascular disease (CVD) in chronic renal failure (CRF) in Malaysia. Global Journal of Health Science. 2011; 3(1): 119 - 27. 
6. Slattery M.L, Wolff RK, Herrick J, Caan, BJ, Samowitz, W. Tumor markers and rectal cancer: Support for an inflammation-related pathway. International journal of cancer. 2009; 125(7): 1698-1704.

7. Wang, F, Arun P, Friedman J, Chen Zh, Van Waes C. Current and potential inflammation targeted therapies in head and neck cancer. Current opinion in pharmacology. 2009; 9(4): 389 - 95.

8. Chua W, Charles KA, Baracos, VE, Clarke SJ. Neutrophil/lymphocyte ratio predicts chemotherapy outcomes in patients with advanced colorectal cancer. British journal of cancer. 2011; 104(8): 1288 95.

9. Kishi, Y, Kopetz S, Chunet YS, Palavecino, Abdalla EK, Vauthey JN. Blood neutrophil-to-lymphocyte ratio predicts survival in patients with colorectal liver metastases treated with systemic chemotherapy. Annals of surgical oncology. 2009; 16(3): 614 - 22.

10. Oh BS, Jang JW, Kwon JH, You CR, Chung KW, Kay CS, et al. Prognostic value of C-reactive protein and neutrophil-to-lymphocyte ratio in patients with hepatocellular carcinoma. BMC cancer. 2013; 13(122): 1- 9.

11. Chen, J, Hong D, Zhai Y, Shen P. Meta-analysis of associations between neutrophil-to-lymphocyte ratio and prognosis of gastric cancer. World journal of surgical oncology. 2015; 13(122): 1 - 9.

12. Zhang, X., Zhang W, Feng LJ. Prognostic significance of neutrophil lymphocyte ratio in patients with gastric cancer: a meta-analysis. PloS one. 2014; 9(11): e111906.

13. Smith RA, Bosonnet L, Raraty M, Sutton R, Neoptolemos JP, Campbell F, et al. Preoperative plateletlymphocyte ratio is an independent significant prognostic marker in resected pancreatic ductal adenocarcinoma. The American Journal of Surgery. 2009; 197(4): 466-72.

14. Nash, G, Turner LF, Scully MF, Kakkar AK. Platelets and cancer. The lancet oncology. 2002; 3(7): 42530 .

15. Hsu J-T, Liao C-K, Le P-H, Chen T-H, Lin C-J, Chen J-S, et al. Prognostic value of the preoperative neutrophil to lymphocyte ratio in resectable gastric cancer. Medicine. 2015; 94(39): 1 - 6.

16. Biglarian A, Hajizadeh, E, Kazemnejad A, Zali, M, et al. Survival analysis of gastric cancer patients using Cox model: a five year study. Tehran University of Medical Sciences. 2009; 67(5).

17. Biglarian A, Hajizade E, Kazennejad A, Zali MR, et al. Postoperative survival prediction in patients with gastric cancer. 2009.

18. Moghimi B, Rajaeifard AR, Tabatabaei HR, Zighami B, Safaei A, Tabei Z. Modeling Survival Analysis Using Cox Model in Gastric Cancer Patients. Iranian Journal of Epidemiology.1987; 3(1):19-24.

19. Laki K. Our ancient heritage in blood clotting and some of its consequences. Annals of the New York Academy of Sciences. 1972; 202(1): 297-307.

20. Yu L, Lv C-V, Yuan A-H, Chen W, Wu A-W. Significance of the preoperative neutrophil-to-lymphocyte ratio in the prognosis of patients with gastric cancer. World Journal of Gastroenterology: WJG. 2015; 21(20): $6280-86$.

21. Chen, N, Liu S, Huang L, Li W, Yang W, Cong T, et al. Prognostic significance of neutrophil-tolymphocyte ratio in patients with malignant pleural mesothelioma: a meta-analysis. Oncotarget. 
2017; 8(34): 57460 - 649.

22. Gu X, Gao X-S, Cui M, Xie M, Peng C, Bai Y, et al. Clinicopathological and prognostic significance of platelet to lymphocyte ratio in patients with gastric cancer. Oncotarget. 2016; 7(31): 49878 - 887.

23. Kemona $\mathrm{H}$. Platelets and inflammatory markers in patients with gastric cancer. Clinical and Developmental Immunology. 2013; 2013.

24. Dogan M, Eren T, Ozdemir N, Cigirgan CL, Zengin. The relationship between platelet-lymphocyte ratio, neutrophil-lymphocyte ratio, and survival in metastatic gastric cancer on firstline modified docetaxel and cisplatinum plus 5 Fluorourasil Regimen: A single institute experience. Saudi journal of gastroenterology: official journal of the Saudi Gastroenterology Association. 2015; 21(5): 320 - 24.

25. He W, Yin C, Guo G, Jiang C, Wang F, Qiu H, et al. Initial neutrophil lymphocyte ratio is superior to platelet lymphocyte ratio as an adverse prognostic and predictive factor in metastatic colorectal cancer. Medical Oncology. 2013; 30(439): 1 - 6.

\section{Tables}

Table 1

Some characteristics of gastric cancer patients

\begin{tabular}{|llll|}
\hline Variable & & Frequency & mean \pm Standard deviation \\
\hline Age & & - & $63.79 \pm 15.03$ \\
\hline Gender & Male & $76(76)$ & - \\
\hline & Female & $24(24)$ & - \\
\hline Location & City & $47(47)$ & - \\
\cline { 2 - 5 } & Village & $53(53)$ & - \\
\hline Education & High school & $79(79)$ & - \\
\hline & Diploma & $21(21)$ & $14.85 \pm 15.17$ \\
\hline Smoking (Year) & - & $1.45 \pm 1.90$ \\
\hline Gastrointestinal Disease (year) & - & & - \\
\hline
\end{tabular}

Table 2

Inflammatory markers of gastric cancer patients studied

\begin{tabular}{|llll|}
\hline Marker & Minimum & Maximum & mean \pm Standard deviation \\
\hline neutrophils to lymphocytes ratio & 0.58 & 16.80 & $3.37 \pm 2.78$ \\
\hline Platelet to lymphocyte ratio & 42.00 & 500.00 & $147.13 \pm 78.93$ \\
\hline
\end{tabular}


Table 3

Survival rate of gastric cancer patients

\begin{tabular}{|ll|}
\hline Property & mean \pm Standard deviation \\
\hline Live & $506.57 \pm 343.29$ \\
\hline Deat & $559.72 \pm 422.28$ \\
\hline Total & $548.56 \pm 405.90$ \\
\hline
\end{tabular}

Table 4

Kaplan-Meier estimator for estimating mean and median survival of gastric cancer patients

\begin{tabular}{|lllll|}
\hline Survive & Estimate & standard error & \multicolumn{2}{l|}{$95 \%$ confidence interval } \\
\cline { 3 - 4 } & & & $\begin{array}{l}\text { Upper } \\
\text { Limit }\end{array}$ & Lower \\
& & & 751 & Limit \\
\hline Mean & 650.70 & 51.10 & 652 & 351 \\
\hline Median & 458.00 & 85.08 & & 319 \\
\hline
\end{tabular}

Table 5

Cox proportional hazard regression model results on gastric cancer patients survival

\begin{tabular}{|llllll|}
\hline $\begin{array}{l}\text { Variable in the } \\
\text { equation }\end{array}$ & $\begin{array}{l}\text { Coefficient } \\
\text { estimation }\end{array}$ & $\begin{array}{l}\text { Standard } \\
\text { error }\end{array}$ & $\begin{array}{l}\text { Significance } \\
\text { level }\end{array}$ & $\begin{array}{l}\text { Relative } \\
\text { risk }\end{array}$ & $\begin{array}{l}\text { 95\% confidence } \\
\text { interval } \\
\text { relative risk }\end{array}$ \\
\hline NLR & 0.166 & 0.042 & $<0.001$ & 1.18 & $1.087-1.284$ \\
\hline PLR & 0.001 & 0.002 & 0.646 & 1.001 & $997-1.004$ \\
\hline
\end{tabular}

Figures 


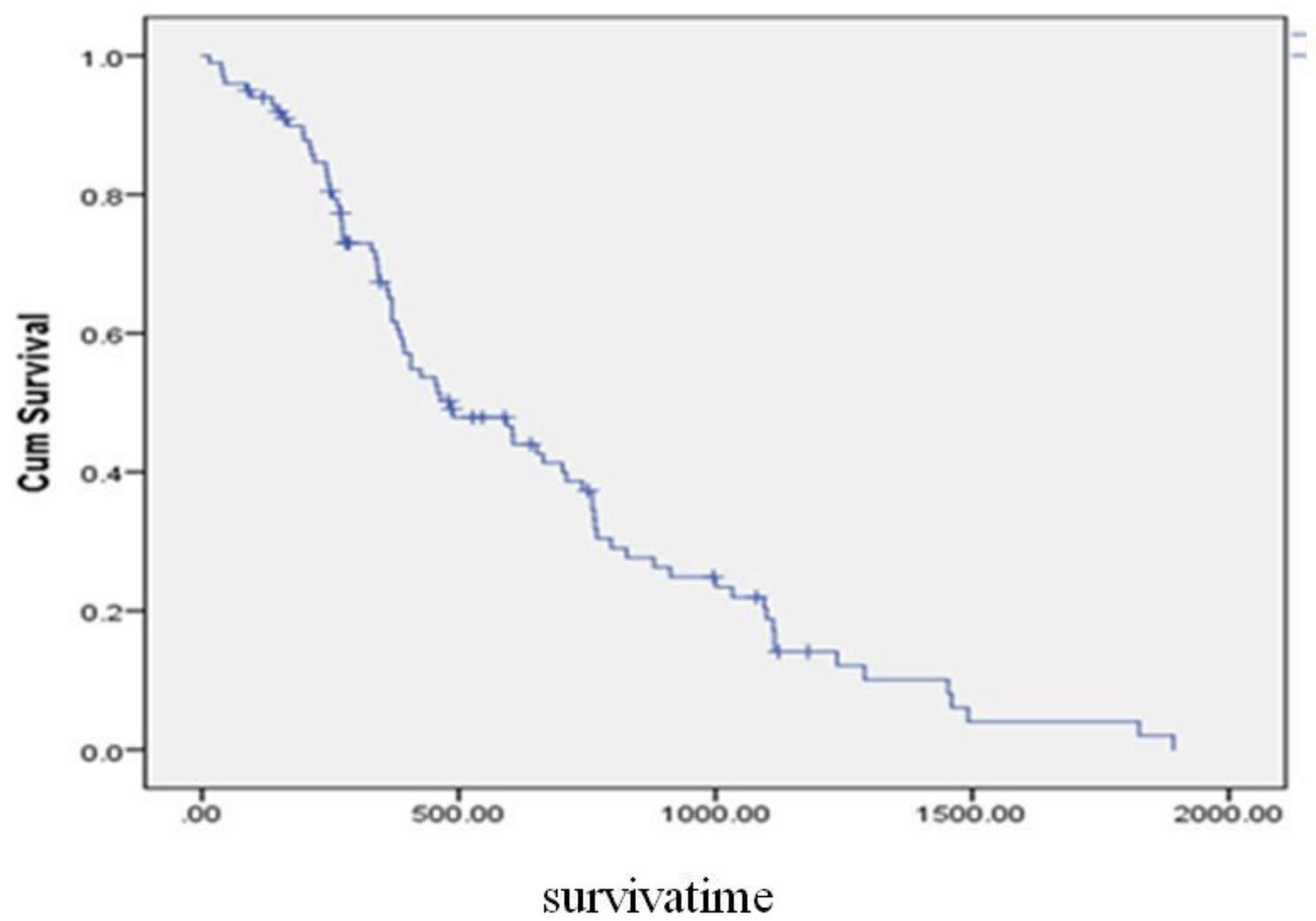

Figure 1

Kaplan-Meier survival function of gastric cancer patients 


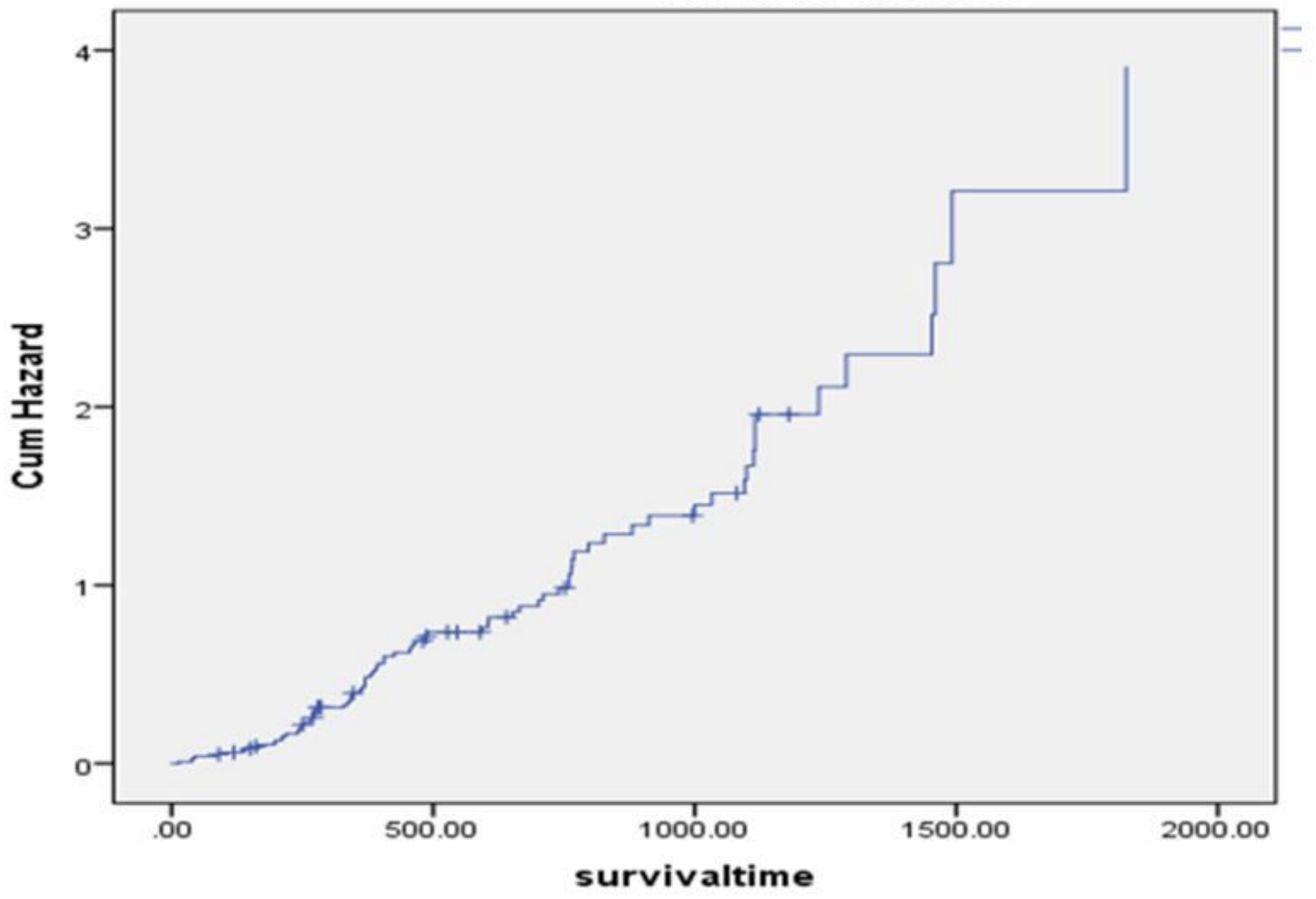

Figure 2

Kaplan-Meier survival function of gastric cancer patients 